\title{
Verification of Signature along with Automated Cheque System
}

\author{
Swathi $\mathbf{J}^{1 *}$, Deepika Sasi ${ }^{2}$, Raseena Rahim ${ }^{3}$, Anu Mariam Alex ${ }^{4}$, Vibesh V Panicker ${ }^{5}$ \\ 1,2,3,4,5 M. Tech Student, APJ Abdul Kalam Technological University, ECE Department, \\ TKM College of Engineering, Karikode, Kollam, Kerala, India. \\ ${ }^{5}$ Assistant Professor, ECE Department, College of Engineering Adoor, Kerala, India. \\ *swathiacer@gmail.com
}

\begin{abstract}
Signature is an unmistakable part for recognizing a person's verification. Indeed, even today an expanding number of exchanges, particularly identified with business and monetary are being approved by means of signs. Subsequently the need have techniques for programmed signature check must be created if legitimacy is to be confirmed and ensured effectively all the time. Ways to deal with signature check fall into two classes as indicated by the securing of the information: Off-line and Electronic acquisition of details. A solid signature confirmation framework is significant piece of law implementation, security control and much business process. It very well may be utilized in numerous applications like cheques, declarations, contracts and so on. The coordinated signature confirmation framework joins information base administration, commotion evacuation and pre-preparing, highlight extraction, learning and check modules. The coordinating model is done and dynamic depends on edge based method that gives close to applications. The framework shows promising outcome. It is planned and executed for robotized confirmation of signature and cheque preparing framework for simple check and investigation of information. Diverse limit esteems are utilized for coordinating relying upon testing and preparing highlights vectors, so we can support the general execution of the framework. It is used for sans cheque and to check whether the cheque is cleared or bounced. For that it varies account number from database and then verifies signature from the master and put the output of matching in percentage. After that amount has been deposited or withdrawn from the respective account
\end{abstract}

Keywords- Image processing, Feature extraction, Noise removal, off- line data and electronic acquisition of details, Threshold values.

\section{INTRODUCTION}

Indeed, even today an expanding number of exchanges, particularly identified with money related and business are being approved through signs. Signature is a distinctive component for individual recognizable proof. Consequently the need to have strategies for programmed signature confirmation must be created if validness is to be checked and ensured effectively all the time. Ways to deal with signature confirmation fall into two classifications as per the procurement of the information: Electronic and Off-line. Electronic information records the movement of the pointer while the signature is created, and incorporates area, and perhaps speed, increasing speed and pen pressure, as elements of time.

Electronic frameworks utilize this data caught during procurement. The dynamic attributes are explicit to every person and adequately steady just as monotonous.
Disconnected information is a 2-D picture of the sign. Handling disconnected is unpredictable because of the nonattendance of stable unique attributes. Here, we tackle the issue in two stages. The principal that the checked sign picture is pre-handled to be reasonable for extricating highlights. At that point the pre-handled picture is utilized to remove significant geometric parameters that can recognize signs of various people.

The following stage includes the utilization of these extricated highlights to check a given picture. The consolation behind this paper is the developing requirement for a phony evidence signature check plot which can ensure most extreme conceivable security from counterfeit signs. The thought behind this paper is likewise to guarantee that the proposed plan can give practically identical and if conceivable preferred execution over effectively settled disconnected signature check plans. 


\section{RELATED WORK}

Mane et al. [1] put forward the idea for fake signature analysis using the signature checking technique. Ferrer et al. [2] studied about disconnected geometric parameters for programmed signature examination utilizing fixed-point number juggling. Jena et al. [3] put forward the progressed Offline signature validation plot utilizing extraction technique for point applications.

\section{METHODOLOGY}

Diagram 1 shows the design and implementation of signature verification and cheque processing. The design helps for better performance, saves time and avoids the chances of falsification.

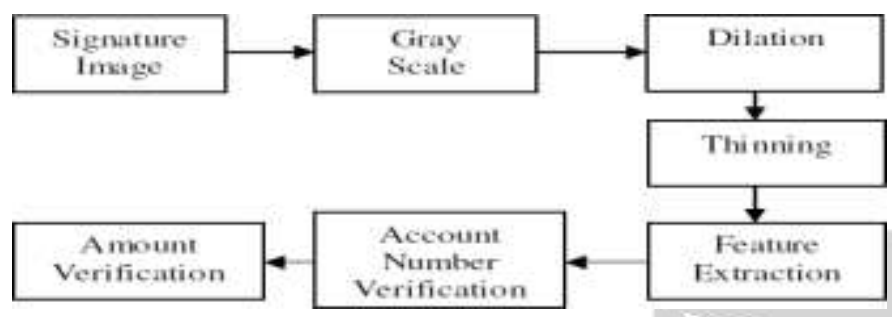

Fig. 1: System Layout

The current work centers around structuring and executing mechanized signature confirmation and cheque handling framework to guarantee preferable execution over recently settled disconnected signature confirmation plan to viably decrease manual work. Pre-preparing brings about a dim scale picture which is then utilized for extraction of the element. At that point, highlights are removed by applying different systems. The training images are obtained using the scanner. The pre-processing operation is applied on these images. The account number and amount verification module is used for verifying the account number and amount using the OCR technique. After this verification process the results are generated to decide whether cheque is cleared or bounced.

This system's configuration and implementation is divided into following modules as -

\subsection{Signature Analysis Module}

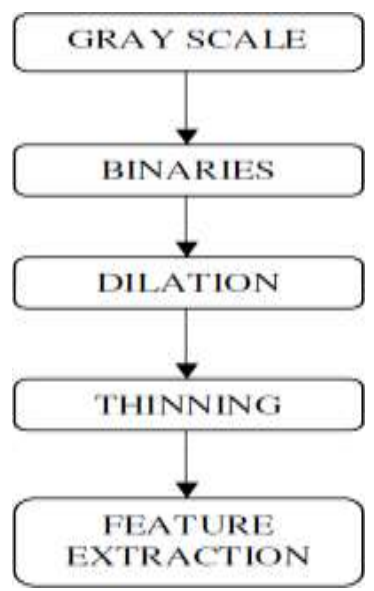

Fig. 2: Signature Analysis Process
This module is used to avoid falsification \& prevents from unauthorized users. In this module the cheque is first scanned using the scanner, the original training set will be used and pre- processed to get suitable for extracting features.

This module is used to avoid falsification \& prevents from unauthorized users. In this module the cheque is first scanned using the scanner, the original training set will be used and pre-processed to get suitable for extracting features.

\subsection{Account Number Analysis Module}

In this model the amount is verified so that the amount to be drawn should be less than the amount deposited. The amount is verified by OCR technique.

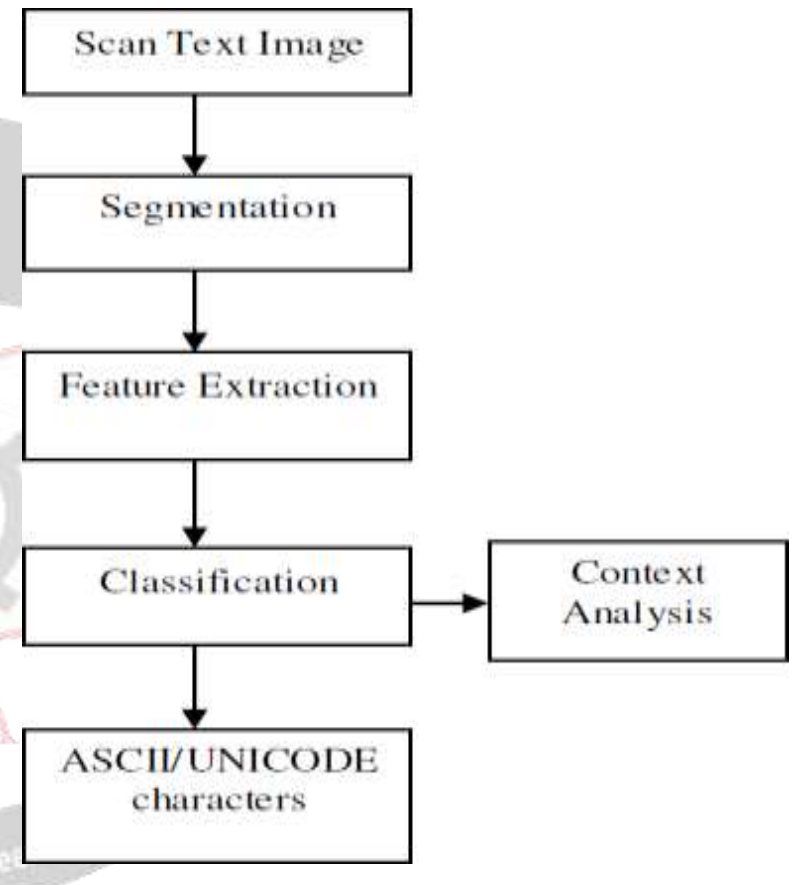

Fig. 3: OCR Steps

Optical Character Recognition is a technique wherein the interpretation from a composed book or examined picture to an ASCII character was completed. The outline of the paper itself may either be machine-printed or written by hand, or the mix of two. Acknowledgment of composed characters is itself a troublesome issue when an adjustment of a similar character occurs because of modification in textual styles or expansion in different types of clamor. Contrast type and design confound the procedure of ID. Subsequently a fruitful answer for character recognizable proof needs to expel the commotion. In the wake of perusing double picture information, smooth out the picture for improved distinguishing proof, effectively remove traits, train the machine and arrange designs. The examined picture of cheque has been utilized for all the tasks. 


\section{SYSTEM OVERVIEW}

\subsection{Classification of Analysis Systems}

The analysis systems can typically be classified into 2 types, depending on the way in which the data is received. They are online and offline signature systems:

\subsubsection{Online Analysis Systems}

The information is gotten by means of sensors utilizing electronic sign plans. The information acquired is typically dynamic information including speed, increasing speed, weight, and tip pressure, angle. These information are normally intra-individual invariant, which implies that for the most part stay unaltered for a solitary individual. This kind of signature check is in this manner profoundly solid and offers a high level of precision. Be that as it may, the fundamental inadequacy in this training is the accessibility and costs engaged with the acquisition of exceptionally delicate instruments used to acquire and investigate information. These imperatives limit the utilization of online recognizable proof. Online recognizable proof can without much of a stretch be reached out to different spaces like unique signature investigation, iris ID, palm print examination and retina examination.

\subsubsection{Offline Analysis Systems}

Framework investigate frameworks require the recovery of uninvolved information. The subtleties wherein the printing is made accessible to various outsider hardware, for example, cameras and printers, in an advanced arrangement. Such methodologies are normally financially savvy. In any case, they don't give the straightforward dynamic information that may have been accumulated from other dynamic applications. Static information acquired from a disconnected signature confirmation strategy might be local, procedural, geometric or numerical.

\subsection{Styles of Falsification}

Different forms of falsification include:

\subsubsection{Spontaneous Falsification}

Spontaneous falsification is performed by a person who doesn't perceive the structure and design of the first signature. (Fig. 4. (b))

\subsubsection{Easy Falsification}

In this kind of fraud, the individual included has a general comprehension of the genuine signature, however he is signing with no training. (Fig 4. (c))

\subsubsection{Professional Falsification}

This style of falsification thinks about adequate consciousness of the first signature and satisfactory time for cautious readiness. Our proposed conspire evacuates unconstrained and simple fraud and along these lines diminishes proficient fabrication to a critical degree. (Fig. 4. (d))

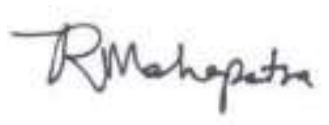

(a)

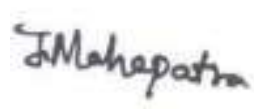

(c)

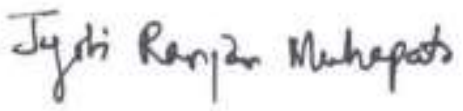

(b)
Fig. 4: Styles of Falsification (a) Original Signature, (b) Spontaneous Falsification, (c) Easy Falsification, (d) Professional Falsification

\subsection{Existing techniques:}

\subsubsection{Arithmetic Fixed Point Process}

This system is centered around geometrical components, which are focused on two vectors portraying the meaning of the envelope and the dissemination of the internal stroke in polar and Cartesian directions.

\subsubsection{Detection and Depiction of Design}

The portrayal is resolved utilizing morphological tasks. To start with, expansion is utilized to decrease signature instability, and afterward a filling technique is utilized to improve the diagram extraction process. At the point when a few articles are distinguished in the wake of filling, even development is done until all items are connected.

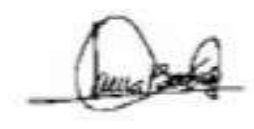

(a)

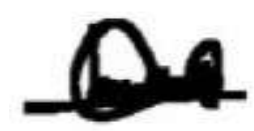

(b)

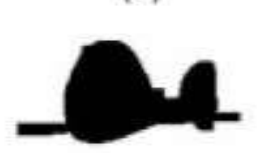

(c)

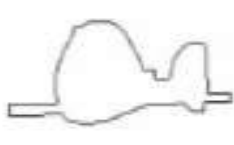

(d)
Fig. 5: (a) Underlying, (b) Enlarged, (c) Completed, (d) Description of signature [1]

\section{RESULT AND DISCUSSIONS}

This work is used to scans the cheque \& tell whether the cheque is bounced or cleared. It verifies the account no from database $\&$ then verifies signature from masters $\&$ puts the output of matching in percentage. Coordinating is completed and dynamic depends on a limit subordinate methodology offering ascend to approach applications. The 
program has created positive advancement. Different edge esteems are utilized for arrangement, in light of the testing and preparing properties of the vectors, in this manner improving the general effectiveness of the technique. The parity is then stored or deducted from the comparing account.

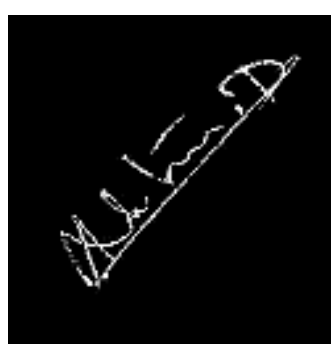

(1)

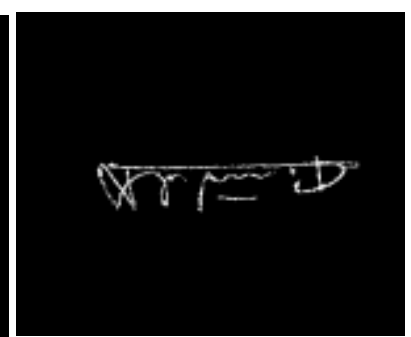

(2)
Diagram 6: (1) Initial Version of Sign (2) Inverted Version

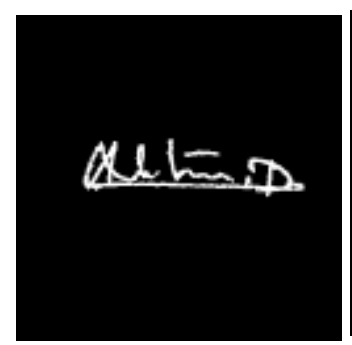

(3)

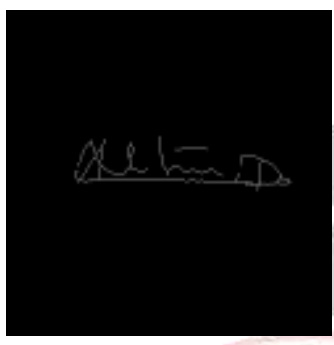

(4)
Diagram 7: (3) Enlarged Version, (4) Thinned Version

\section{CONCLUSIONS}

It might be seen in different manners, for example, tests, licenses, contracts, and so forth. A protected program of signature confirmation is an indispensable part of law requirement, get to the board and different business forms. The coordinated sign confirmation program involves database control, commotion decrease and pre-handling, recovery, learning and testing modules. The objective of further research is to distinguish a new, proficient capacity vector which will bring about an insignificant deviation for every signature occasion which might be made so as to additionally expand the accuracy of the program.

\section{REFERENCES}

[1] Kishore T. Mane, Vandana G. Pujari, "Signature Matching with Automated Cheque System", International Conference on Intelligent Systems and Signal Processing (ISSP), 2013.

[2] MigualA. Ferrer, Jesus B. Alonso and Carlos M. Travieso, "Off-line Geometric Parameters for Automatic Signature Verification Using Fixed-Point Arithmetic", IEEE Tran. on Pattern Analysis and Machine Intelligence, vol.27, no.6, June 2005.

[3] Debasish Jena, BanshidharMajhi, Saroj Kumar Panigrahy, Sanjay Kumar Jena"Improved Offline Signature Verification Scheme Using Feature Point ExtractionMethod".

[4] T.Y. Zhang, C.Y.Ceun "A fast algorithm for Thinning
Digital Patterns". Communications of ACM, March 1998 Concepts of Image Processing.

[5] S. Mori et al. " Historical Review of OCR Research and Development”, IEEE, 80, no 7, pp. 1029-1058, July 1992.

[6] A.A Chaudhary, E.A.S.Ahamad, S.Hossain, C.M.Rahman, "OCR of Bangla Character Using Neural Network: A better Approach“. $2^{\text {nd }}$ International Conference on Electrical Engineering (ICEE 2002), Khuln Bangaldesh.

[7] Utpal Garain and Bidyut B. Chaudhary, "Segmentation of Touching Character in Printed Devnagari and Bangla Script Using Fuzzy Multi factorial Analysis”, IEEE Transaction on System, Man and Cybernetics-Part C: Applications and Reviews, 32, November 2002. Page(s): 449-459.

[8] Alexander.J.Faaborg, "Using Neural Network to Create an Adaptive Character Recognition System".

[9] B. B. Chaudhuri and U. Pal., "Skew angle Detection of Digitized Indian script Documents". Pattern Analysis and Machine Intelligence, IEEE Transactions on Volume 19, Issue 2, Feb. 1997 Page(s):182-186.

[10] D Amato., "A document skew detection method using runlength encoding and the Hough transform", Pattern Recognition, 1990. IEEE Proceedings., 10th International Conference on Volume 1, 16-21 June 1990 Page(s):464-468 vol.1.

[11] Nakamura.G. "A thinning algorithm for digital figures of characters". Image Analysis and Interpretation, 2000. Proceedings. 4th IEEE Southwest Symposium 2-4 April 2000 Page(s):83-87. 